\title{
Monte Carlo Simulation of Liquid-Crystal Alignment and of Chiral Symmetry-Breaking
}

\author{
Jianling Xu and Robin L. B. Selinger \\ Physics Department, Catholic University of America, Washington, DC 20064 \\ Jonathan V. Selinger and R. Shashidhar \\ Center for Bio/Molecular Science and Engineering, Naval Research Laboratory, Code 6900, \\ 4555 Overlook Avenue, SW, Washington, DC 20375
}

(June 11, 2001)

\begin{abstract}
We carry out Monte Carlo simulations to investigate the effect of molecular shape on liquid-crystal order. In our approach, each model mesogen consists of several soft spheres bonded rigidly together. The arrangement of the spheres may be straight (to represent uniaxial molecules), Z-shaped (for biaxial molecules), or banana-shaped (for bent-core molecules). Using this approach, we investigate the alignment of the nematic phase by substrates decorated with parallel ridges. We compare results for wide and narrow ridge spacing and examine local order near the substrates, and show that our results are consistent with the predictions of Landau theory. We also investigate chiral symmetrybreaking in systems of bent-core molecules. We find a chiral crystalline phase as well as a nonchiral smectic-A phase, but not a chiral smectic-C phase.

PACS numbers: $61.30 . \mathrm{Cz}, 61.30 . \mathrm{Hn}, 64.70 . \mathrm{Md}$
\end{abstract}

\section{INTRODUCTION}

Molecular simulations have emerged as an important tool for investigating liquid crystals. The goal of the simulations is to relate the microscopic structure of molecules to the macroscopic properties of liquid-crystal phases. To do this, researchers begin with some assumptions about molecular structure, simulate a large number of interacting molecules, and determine the large-scale order of the resulting system as a function of temperature, applied fields, and other variables. This technique provides insight into the origin of liquid-crystal phase transitions and textures. It also assists in the development of new liquid-crystal systems for technological applications.

Researchers have used several approaches for molecular simulations of liquid crystals. On the most microscopic scale, some studies begin with the structure of liquid-crystal molecules in atomistic detail, and let the molecules interact through a potential derived from the sum of interatomic interactions. approach is that it can simulate actual experimental materials and can show how macroscopic properties change with slight variations in the chemical structure. The disadvantage is that this approach is computationally intensive, and hence it can only be done for relatively small systems, which may not be large enough to show all the important features of liquid-crystal ordering. Accuracy of classical potentials can also be an issue.

Because of these limitations, many other studies begin with greatly simplified models of molecular structures and interactions, which require less computer time and hence allow larger system sizes. Some studies represent molecules as cylinders or spherocylinders, which inter- act through a hard or soft excluded-volume repulsion. 3 国 Other studies represent molecules as ellipsoids interacting through the Gay-Berne potential, an anisotropic generalization of the Lennard-Jones poteptial. 6 a related approach uses the Corner potential.9 To model more complicated molecular structures, further studies represent molecules as composites of two Gay-Berne ellipsoids. 10 Soft-sphere potentials can also be joined together in a linear chain to represent mplecules. Such molecules can be rigid 11 or semiflexible.12.13

As an alternative approach, we model liquid-crystal molecules as a composite of soft spheres bonded rigidly into a fixed shape, with no intramolecular flexibility. This approach is particularly useful for two reasons. First, the intermolecular interaction can be calculated at low computational cost, thus allowing the simulation of large systems. Second, the spheres may be bonded into any shape to represent molecular structures of any symmetry. These features allow us to explore the complex relationship between molecular shape and phase behavior, without undertaking full-scale atomistic simulations. The approach thus represents a compromise between the simplest and most complex models used in liquid crystal simulation.

In a recent paper, 4 we used this approach to simulate smectic liquid crystals. Our goal was to model the electroclinic effect, in which an applied electric field induces molecular tilt in the smectic-A phase. For that reason, we considered model molecules with the structure shown in Fig. 1(a). The overall shape is a biaxial zig-zag shape like the letter $\mathrm{Z}$, and a transverse electric dipole moment makes the molecule chiral. This shape was motivated by the fact that many real liquid-crystal molecules exhibiting the electroclinic effect have this general structure. 
Indeed, the biaxiality of this structure should be important for the electroclinic effect because a tilted smectic phase is biaxial. Our simulation results confirmed that the degree of biaxiality or obliqueness in molecular shape is crucial for spontaneous molecular tilt in the smectic$\mathrm{C}$ phase and for induced molecular tilt in the smectic- $\mathrm{A}$ phase.

The purpose of our current study is to explore this simulation method further by applying it to two different problems in the physics of liquid crystals. First, we consider the alignment of a nematic liquid crystal by a ridged surface. Second, we explore chiral symmetry-breaking in the smectic phases of a bent-core (or banana-shaped) liquid crystal. In each case, we construct model mesogens consisting of soft spheres bonded rigidly together with an appropriate shape. Our results for these two problems shows that this simulation method is a versatile approach for modeling different types of liquid-crystal ordering.

The first problem that we consider, the surface alignment of a nematic phase, is a classic problem in liquidcrystal science apd is fundamental to all applications of liquid crystals 15 .1 Liquid-crystal cells are constructed so that the surfaces favor alignment of the molecules in a particular direction, which may be planar (in a particular direction within the surface plane), homeotropic (normal to the surface), or between these extremes. An applied electric field can cause the molecules in the interior of the cell to tilt away from the alignment favored by the surfaces. Thus, the competition between surface alignment and an applied field allows the molecules in the cell to be switched between different orientations, changing the optical properties of the cell. Key questions in designing new surfaces to optimize liquid-crystal alignment are: What type of surface is needed to align liquid-crystal molecules? How far into a cell does the alignment persist?

To address these questions, we perform Monte Carlo simulations of nematic alignment, using the simulation approach discussed above. For the model molecules, we would like to construct a rigid uniaxial rod-like structure, which is known to give a nematic phase at high enough densities. For that reason, we use seven spheres bonded together in a straight line, as shown in Fig. 1(b). For the aligning surface, we would like to model a rubbed polymer substrate, which has parallel ridges that give planar alignment of the liquid crystal. We use a flat surface decorated with regularly spaced elevated ridges, each composed of spheres fixed in a row, as shown in Fig. 2. Our simulation results show that the nematic director aligns with the ridges for all ridge spacings studied. The nematic order parameter near the substrate increases as the ridge spacing is reduced, indicating that narrowly spaced ridges on the substrate suppress director fluctuations. However, the correlation length associated with the drop-off of nematic order away from the substrate is independent of ridge spacing, and is a characteristic of the bulk nematic phase.

Our second problem, chiral symmetry-breaking in sys- tems of bent-core molecules, concerns a new type of smectic liquid crystal. In recent years, researchers have synthesized liquid-crystal molecules with a bent or "bananashaped" core 1719 The individual molecules are not chiral. In the bulk or in freely suspended films, the molecules form smectic phases. In the smectic-A phase, the molecules are on average normal to the smectic layers, and hence the phase is not chiral. However, when the material is cooled into a smectic-C phase, the molecules can tilt with respect to the layers in two inequivalent ways that are mirror images of each other. For that reason, the system undergoes a chiral symmetry-breaking transition, in which it forms right- and left-handed chiral domains 1719 There are actually several different smectic$\mathrm{C}$ phases, depending on how the domains of alternate chirality and alternate tilt pack together. Key questions for the theory of these systems are: What type of intermolecular interaction drives the chiral symmetrybreaking transition? How do the molecules pack together in the achiral and the chiral phases?

To investigate these issues, we perform Monte Carlo simulations of bent-core liquid crystals, again using the same simulation approach. In this case, we construct model molecules by arranging seven soft spheres in a rigid bent-core shape, as shown in Fig. 1(c). In the simulations, we find that a bulk system of these molecules forms a nonchiral smectic-A phase at high temperatures, and a chiral tilted crystalline phase at low temperatures. The chiral symmetry-breaking transition is driven by a favored packing of the molecules, which can fit together more efficiently in either a right- or a left-handed state than in a symmetric state. Thus, at least in this model, the steric interaction of rigid molecules is sufficient for chiral symmetry-breaking; neither molecular flexibility nor dipole-dipole interactions are necessary. Although this system forms a chiral tilted phase with three-dimensional crystalline order, it does not form a chiral smectic-C phase with only one-dimensional positional order. We believe that the simultaneous onset of chiral order and crystalline order is an artifact of our particular choice of molecular shape, because the same type of chiral order can occur in either a crystalline or a smectic-C phase.

\section{SURFACE ALIGNMENT OF NEMATIC PHASE}

To simulate a nematic phase, we use the rod-like model molecule shown in Fig. 1(b). Each molecule is composed of seven spherical particles, or interaction sites, fixed rigidly in a straight line, with no intramolecular degrees of freedom. The sphere-sphere interaction potential is the truncated Lennard-Jones potential cut off at its minimum so there is no attractive tail, also known as the Weeks-Chandler-Andersen potential, 20 


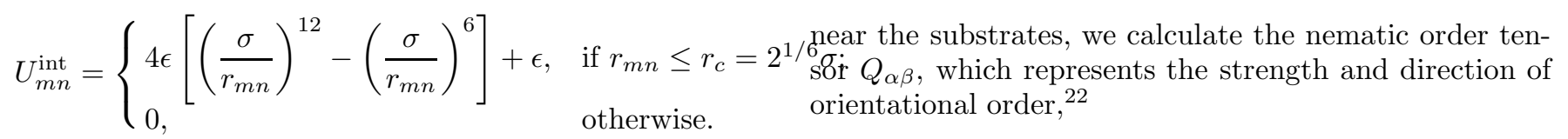

where $r_{m n}=\left|\mathbf{r}_{m}-\mathbf{r}_{n}\right|$ and $m$ and $n$ are the sphere indices referring to different molecules. In this way, the steric interactions between molecules are represented via a short-range repulsive potential, with no attractive or electrostatic interactions.

The simulation cell consists of 5,070 molecules in a three-dimensional rectangular box of size $35 \times 35 \times 41$, where lengths are measured in units of the Lennard-Jones parameter $\sigma$, and each molecule is seven units long and one unit wide. The system has periodic boundary conditions along $x$ and $y$, and is confined in the $z$ direction between parallel substrates.

Each substrate consists of a soft repulsive wall that interacts with the spherical particles in each molecule using the same WCA potential. Each substrate is decorated with regularly spaced elevated ridges composed of spheres fixed in parallel rows along the wall, as shown in Fig. 2. Each of these spheres is identical to the spheres making up the liquid-crystal molecules, and again has the same WCA interactions. Thus, interactions between the liquid crystal and the wall are essentially steric in nature, with no attractive or electrostatic terms.

Each wall has an integer number of ridges, spaced evenly. We make the ridge pattern the same for the top and the bottom substrates, so that the nematic is not twisted, having the same preferred planar orientation on both substrates. However we slightly shift the ridge patterns so that the substrates do not have an exact mirror symmetry.

We perform Monte Carlo (MC) simulations at the fixed temperature $k_{B} T=5.0$, well above the nematic-smecticA transition point, which is about 3.5, measured in units of the Lennard-Jones parameter $\epsilon$. The density is held fixed at $\rho=0.7$ spheres/unit volume. In preliminary simulations, we either randomize the initial molecular orientations or initialize them in a homeotropic orientation, aligned normal to the substrates. We find that either of these initial conditions leads to a slow ordering process, which eventually gives rise to an aligned planar structure, with the nematic director parallel to the ridges on the substrates. After determining the preferred orientation, we perform further simulations with the molecules initialized in the preferred planar orientation, with the molecules aligned parallel to the ridges and the centerof-mass positions randomized.

Each MC step consists of three attempted translations (along $x, y$, and $z$ ) and two attempted rotations (in $\theta$ and $\phi)$ per molecule, with appropriate sampling in the polar angle.21 We carry out 100,000 MC steps to reach equilibrium and then an additional 200,000 MC steps to collect data.

To measure the spatial variation of liquid-crystal order

$$
Q_{\alpha \beta}=\left\langle\frac{1}{N} \sum_{j=1}^{N}\left(\frac{3}{2} n_{j \alpha} n_{j \beta}-\frac{1}{2} \delta_{\alpha \beta}\right)\right\rangle
$$

where $\mathbf{n}_{j}$ is the director along the axis of molecule $j$ and $N=5070$ is the number of molecules. For any $z$, the largest eigenvalue of $Q_{\alpha \beta}(z)$ represents the scalar nematic order parameter $S(z)$. The corresponding eigenvector identifies the local direction of alignment.

We evaluate $Q_{\alpha \beta}$ as a function of distance from the substrate by dividing the system into fifty layers and calculating $Q_{\alpha \beta}$ in each layer. Because the number of molecules in each layer is relatively small, around 100, we must average over time to get a statistically meaningful sample. Accordingly, we average over a series of 100 independent configurations separated in time by 2000 MC steps each.

We perform simulations using substrates with three different ridge spacings, but with the same bulk density $\rho=0.7$ spheres/unit volume, the same temperature $k_{B} T=5.0$, and the same number of molecules $N=5070$ (a total of 35,490 particles, plus those decorating the substrate). The number of ridges on the substrates is chosen as 1,2 , and 7 , with spacing between ridges of $35,17.5$, and 5 length units, respectively (taking periodic boundary conditions into account). The box dimensions are set at $H_{x}=35, H_{y}=35$, and $H_{z} \approx 41$, with $H_{z}$ adjusted slightly to make the bulk densities the same for all three systems, as the total number of particles in the box is slightly increased by the addition of extra ridges.

In each case we find that the nematic director throughout the cell aligns parallel to the ridges. Figure 3 shows the nematic order parameter $S(z)$ vs. $z$ for all three systems, with one substrate at $z=0$ and the other at $z=H_{z}$. The bulk order parameter is about the same in all cases, but the order near the substrate varies with ridge spacing. The narrowest ridge spacing gives the largest enhancement in local order, while the widest ridge spacing gives the least enhancement. Figure 3 also shows that the correlation length associated with nematic order near the interface is independent of the ridge spacing. In other words, no matter how densely spaced the substrate ridges, the thickness of the ordered interface does not change. We fit the data in Fig. 3 to the form

$$
S(z)=S_{0}+A\left(e^{-z / \xi}+e^{-\left(H_{z}-z\right) / \xi}\right),
$$

where $S_{0}$ is the bulk order parameter value, $A$ is the amplitude of enhancement near the substrates, and $\xi$ is the associated correlation length. Using the least-squares algorithm, we fit the data for all three ridge spacings. Figure 3 shows an example of the fit for the ridge spacing of 5 units, and Table I gives the fit parameters.

Our results can be understood through an analytical calculation using a Landau free energy functional, which 
is similar to the model in Ref. 23. We assume that the nematic order parameter takes the form,

$$
S(z)=S_{0}+S_{1}(z)
$$

where $S_{0}$ is the bulk value and $S_{1}(z)$ is the surfaceinduced contribution. Near a single aligning substrate, the free energy can be written as

$$
F=\int d z\left[\frac{1}{2} K\left(\frac{d S}{d z}\right)^{2}+\frac{1}{2} a\left(S(z)-S_{0}\right)^{2}\right]-h S(z=0) .
$$

where $a$ and $K$ are bulk parameters dependent on temperature and density. The term $-h S(z=0)$ favors enhanced nematic order at the substrate, and one expects the anchoring strength $h$ to vary with substrate structure.

Minimizing the free energy functional gives

$$
S(z)=S_{0}+A e^{-z / \xi}
$$

where $A=h / \sqrt{K a}$ and $\xi=\sqrt{K / a}$. These equations show that the amplitude $A$ is a surface parameter, which depends on $h$ as well as $K$ and $a$ and thus varies with substrate structure. By contrast, the correlation length $\xi$ is a bulk parameter, which depends only on $K$ and $a$ and thus varies with temperature and density but is independent of substrate structure. This is consistent with the simulation results for $A$ and $\xi$ in Table I.

Apart from the enhanced nematic order near the substrates, the simulations also show a local tendency to smectic ordering near the substrates, even though the temperature is well above the bulk nematic-smectic transition. Figure 4 shows a snapshot of the molecular configuration near one substrate, for the ridge spacing of 5 units. Clearly the molecular density is not uniform. Instead, there are local regions of incipient molecular layering in the direction along the ridges. This local layering suggests that the system is near a surface nematicsmectic transition. The tendency to smectic ordering in a nematic close to a surface with planar orientation has also been studied theoretically in the case of a bare substrate.24 In that system, as in our system, the wall suppresses local director fluctuations, giving rise to a more ordered state, just as an applied electric field can drive a nematic-smectic transition.14

In experiments on nematic alignment by buffed surfaces, it has sometimes been observed that a nematic aligns in a direction different from, or even perpendicular to, the buffing axis 25 While our simulations did not show that kind of ordering, our results lead us to consider possible explanations. One is that the local smectic order next to the substrate could form with the layers parallel to the ridges, and with the molecules oriented perpendicular to the ridges or at some degree of tilt. It is possible that a wider ridge spacing, a different molecular shape, or a temperature closer to the nematic-smectic transition could produce such effects in a simulation like ours. Another likely explanation is that electrostatic or attractive interactions play an important role in determining the preferred orientation. These effects have not been considered in the present model but could be introduced in future work.

\section{CHIRAL SYMMETRY-BREAKING IN SYSTEMS OF BENT-CORE MOLECULES}

As discussed in the Introduction, recent experimental studies have found that liquid crystals composed of certain achiral molecules can undergo a spoptaneous symmetry-breaking and form chiral domains 1719 These "bent-core" or "banana-shaped" molecules form layered structures similar to those observed in ferroelectric smectic liquid crystals, with the molecular axis tilted with respect to the layer normal. Each molecule has a net dipole moment which defines a polar axis perpendicular to the long axis of the molecule. The chirality of the tilted phase is determined by the spatial relationship of the layer normal, the tilt direction, and the polar axis; these can be arranged in either right- or left-handed fashion. The two arrangements are degenerate in energy, leading to the formation of right- and left-handed domains with equal probability.

We perform Monte Carlo simulations to investigate what type of intermolecular interaction can drive the chiral symmetry-breaking transition, and how the molecules pack together in the achiral and chiral phases. In these simulations, we use the same approach discussed above for simulations of nematic alignment, but we change the model molecular structure in order to represent bent-core molecules. The model structure consists of seven spheres arranged in a rigid bent-core shape, like the letter $\mathrm{V}$, as shown in Fig. 1(c). The bend angle between the two straight segments of the molecule is $140^{\circ}$. As in the earlier simulations, the molecules interact through the soft repulsive sphere-sphere interaction of Eq. (11). We neglect dipole-dipole interactions in order to see what ordering is induced by purely steric interactions.

To characterize the magnitude and sign of chirality of the system, we introduce a chiral order parameter $\chi_{i}$ for each molecule $i$. In a smectic phase, let the unit vector $\hat{\mathbf{m}}_{i}$ represent the local layer normal, $\hat{\mathbf{n}}_{i}$ represent the long axis of the molecule, and $\hat{\mathbf{p}}_{i}$ represent the molecular dipole moment. The chiral order parameter can then be defined as

$$
\chi_{i}=\left[\left(\hat{\mathbf{m}}_{i} \times \hat{\mathbf{n}}_{i}\right) \cdot \hat{\mathbf{p}}_{i}\right]\left[\hat{\mathbf{m}}_{i} \cdot \hat{\mathbf{n}}_{i}\right] .
$$

Note that this order parameter is invariant under the two symmetry operations $\hat{\mathbf{m}}_{i} \rightarrow-\hat{\mathbf{m}}_{i}$ and $\hat{\mathbf{n}}_{i} \rightarrow-\hat{\mathbf{n}}_{i}$, but not under $\hat{\mathbf{p}}_{i} \rightarrow-\hat{\mathbf{p}}_{i}$, which is not a symmetry of the system. The average chiral order parameter is then

$$
\bar{\chi}=\frac{1}{N} \sum_{i=1}^{N} \chi_{i} .
$$


That average order parameter can be compared with the rms chiral fluctuations

$$
\chi_{\mathrm{rms}}=\left[\frac{1}{N} \sum_{i=1}^{N}\left(\chi_{i}-\bar{\chi}\right)^{2}\right]^{1 / 2} .
$$

We simulate 600 molecules in a rectangular box with periodic boundary conditions. We choose the height of the box so that the molecules can form four smectic-like layers. The density is held fixed at $\rho=0.7$ spheres per unit volume. At the temperature $k_{B} T=5.0$, the systems forms a chiral crystalline phase. A snapshot of this phase is shown in Fig. 5. In equilibrium, the average chiral order parameter is $\bar{\chi}=0.24$, while the rms average chiral fluctuations are $\chi_{\mathrm{rms}}=0.06$. The average chiral order parameter is much greater than the chiral fluctuations, which means the system is in a well-defined chiral state. This spontaneous chiral ordering can be seen in the snapshot, which shows the molecules tilting in a way that breaks reflection symmetry.

The system shown in Fig. 5 has a large spontaneous tilt in each layer, but the direction of the tilt appears to vary randomly from layer to layer. The coupling between adjacent layers due to excluded volume interactions is apparently too weak to order the tilt directions. A similar variation in the tilt from layer to layer was found in the smectic-C phase of similar molecules 14 Although the tilt directions vary, the chiral order parameter has the same sign in each layer, indicating that the simulation cell represents a domain of uniform chirality.

The structure of a single layer, shown in Fig. 5(b), shows that the system has periodic order with some disordered regions, so it is a crystalline rather than a smectic phase. When we raise the temperature or lower the density of this system, the tilt vanishes and we find a stable untilted smectic-A phase. We do not observe any tilted chiral smectic-C phase. In this system, the onset of chiral order apparently occurs together with the onset of crystalline order.

The observation of a chiral phase, even though it is crystalline rather than smectic, answers the basic questions raised above. First, the simulations show that the steric interaction of rigid molecules is sufficient to drive the chiral symmetry-breaking transition. Because we neglect dipole-dipole interactions in the simulations, we see that they are not necessary for this transition. Furthermore, because we completely suppress intramolecular degrees of freedom, we see that chiral ordering can occur in achiral materials without the involvement of chiral excited molecular states. Second, the snapshot shows how the molecules pack together in the chiral phase. We see that the molecules tilt so that the spheres of one molecule fit into the spaces between the spheres of adjacent molecules. Thus, a tilted chiral state with either handedness is a more efficient packing of neighboring molecules than an untilted achiral state. This shows an explicit mechanism for chiral symmetry-breaking in a system with purely steric interactions.
In fact, the "bumpy" nature of molecular shape may play a crucial role in favoring the tilted geometry, via the details of molecular packing. In our model, two parallel molecules do not pack together efficiently unless they are tilted enough to allow the close packing of the component spheres. A bent-core molecule composed of two spherocylinders has also been simulated, 26 but it does not show a chiral symmetry-breaking transition, presumably because close packing of spherocylinders does not require molecular tilt.

We do not think that there is any fundamental connection between the onset of chiral order and the onset of crystalline order. The same type of chiral order described by the order parameter of Eq. (7) can occur in either a crystalline or a smectic- $\mathrm{C}$ phase. The fact that we do not observe a chiral smectic- $\mathrm{C}$ phase is probably an artifact of the particular molecular structure that we have simulated. To test how sensitively the phase behavior depends on molecular structure, future simulations should make slight variations on the molecular structure. In general, a less sharp bend-angle will favor a smectic phase over a crystalline phase at a given temperature and density. A bump strategically placed at the center of the bent-core molecule will change the packing geometry and might favor twist (of either handedness) over parallel packing. Such a bump can be formed by increasing the interaction distance of the central sphere, or by adding an extra sphere to the molecule. The inclusion of dipoledipole interactions could also destabilize the crystal and favor the formation of a smectic phase.

In summary, we have extended a Monte Carlo simulation method, originally developed for simulations of the electroclinic effect, to model surface alignment of nematic liquid crystals and chiral symmetry-breaking in bent-core liquid crystals. The results give insight into the local molecular arrangements that lead to both of these macroscopic effects. These two studies show that this simulation method is a versatile technique for investigating a wide range of ordering phenomena in liquid crystals.

\section{ACKNOWLEDGMENTS}

This research was supported by the Office of Naval Research and the Naval Research Laboratory.

${ }^{1}$ M. A. Glaser, R. Malzbender, N. A. Clark, and D. M. Walba, J. Phys.: Condens. Matter 6, A261 (1994); Molecular Simulation 14, 343 (1995).

${ }^{2}$ M. A. Glaser, in Advances in the Computer Simulations of Liquid Crystals, C. Zannoni and P. Pasini, editors (Kluwer, Dordrecht, 1999), p. 263. 
${ }^{3}$ A. Galindo, G. Jackson, and D. J. Photinos, Chem. Phys. Lett. 325, 631 (2000).

${ }^{4}$ Z. Dogic, D. Frenkel, and S. Fraden, Phys. Rev. E 62, 3925 (2000).

${ }^{5}$ M. S. Al-Barwani and M. P. Allen, Phys. Rev. E 62, 6706 (2000).

${ }^{6}$ J. G. Gay and B. J. Berne, J. Chem. Phys. 74, 3316 (1981).

${ }^{7}$ M. A. Bates and G. R. Luckhurst, J. Chem. Phys. 110, 7087 (1999).

${ }^{8}$ R. Berardi and C. Zannoni, J. Chem. Phys. 113, 5971 (2000).

${ }^{9}$ H. Zewdie, J. Chem. Phys. 108, 2117 (1998).

${ }^{10}$ R. Memmer, H. G. Kuball, and A. Schönhofer, Liquid Crystals 19, 749 (1995); Molecular Physics 89, 1633 (1996).

${ }^{11}$ G. V. Paolini, G. Ciccotti, and M. Ferrario, Molec. Phys. 80, 297 (1993).

${ }^{12}$ F. Affouard, M. Kröger, and S. Hess, Phys. Rev E. 54, 5178 (1996).

13 J. C. Lee, J. Chem. Phys. 113, 6943 (2000).

${ }^{14}$ J. Xu, R. L. B. Selinger, J. V. Selinger, B. R. Ratna, and R. Shashidhar, Phys. Rev. E 60, 5584 (1999).

${ }^{15}$ L. M. Blinov and V. G. Chigrinov, Electrooptic Effects in Liquid Crystal Materials (New York, Springer-Verlag, 1994), chapter 3

${ }^{16}$ M. G. Tomilin, J. Opt. Tech. 64, 452 (1997).

17 T. Niori, T. Sekine, J. Wanatabe, T. Furukawa, and H. Takezoe, J. Mater. Chem. 6, 1231 (1996).

${ }^{18}$ D. R. Link, G. Natale, R. Shao, J. E. Maclennan, N. A. Clark, E. Körblova, and D. M. Walba, Science 278, 1924 (1997).

${ }^{19}$ G. Heppke and D. Moro, Science 279, 1872 (1998).

20 J. D. Weeks, D. Chandler, and H. C. Andersen, J. Chem. Phys. 54, 5237 (1971); D. Chandler, J. D. Weeks, and H. C. Andersen, Science 220, 787 (1983).

${ }^{21}$ M. P. Allen and D. J. Tildesley, Computer Simulation of Liquids (Oxford, Clarendon Press, 1987), chapter 4, page 132.

${ }^{22}$ P. M. Chaikin and T. C. Lubensky, Principles of Condensed Matter Physics (Cambridge, Cambridge University Press, 1995), page 168.

${ }^{23}$ P. Sheng, Phys. Rev. A 26, 1610 (1982).

${ }^{24}$ A. Poniewierski and A. Samborski, Phys. Rev. E 51, 4574 (1995).

${ }^{25}$ D.-S. Seo, K. Muroi, T. Isogami, H. Matsuda, and S. Kobayashi, Jpn. J. Appl. Phys. 31, 2165 (1992).

${ }^{26}$ Y. Lansac, P. K. Maiti, M. A. Glaser, and N. A. Clark, Bull. Am. Phys. Soc. 46, 689 (2001).

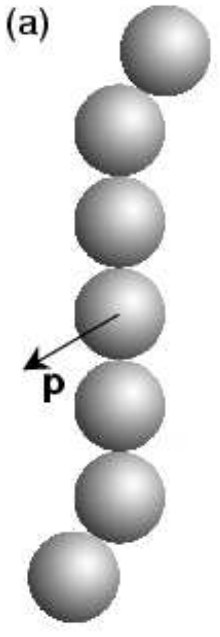

(b)
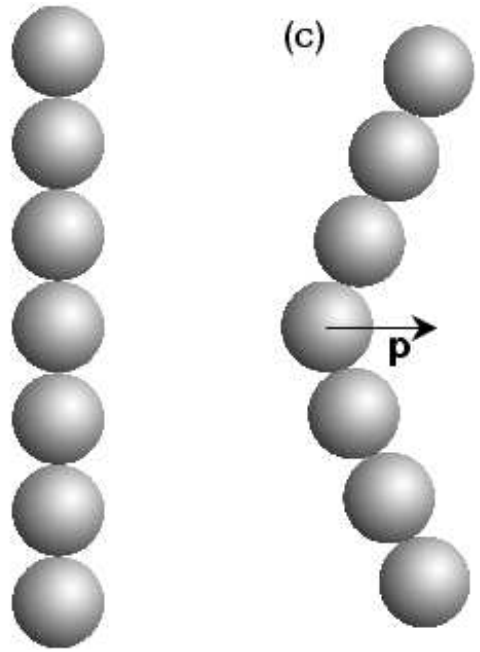

FIG. 1. Structure of the model molecules discussed in this paper: (a) Biaxial Z-shaped molecules, used in simulations of the electroclinic effect.14 The transverse dipole moment $\mathbf{p}$ makes these molecules chiral. (b) Uniaxial rod-like molecules, used in simulations of surface alignment of the nematic phase. (c) Bent-core (banana-shaped) molecules, used in simulations of chiral symmetry-breaking. Because the dipole moment $\mathbf{p}$ is in the molecular plane, the molecules are not chiral.

\begin{tabular}{|lllllll|}
\hline 8 & 8 & 8 & 8 & 8 & 8 & 8 \\
8 & 8 & 8 & 8 & 8 & 8 & 8 \\
8 & 8 & 8 & 8 & 8 & 8 & 8 \\
8 & 8 & 8 & 8 & 8 & 8 & 8 \\
8 & 8 & 8 & 8 & 8 & 8 & 8 \\
8 & 8 & 8 & 8 & 8 & 8 & 8 \\
8 & 8 & 8 & 8 & 8 & 8 & 8 \\
8 & 8 & 8 & 8 & 8 & 8 & 8 \\
8 & 8 & 8 & 8 & 8 & 8 & 8 \\
8 & 8 & 8 & 8 & 8 & 8 & 8 \\
8 & 8 & 8 & 8 & 8 & 8 & 8 \\
8 & 8 & 8 & 8 & 8 & 8 & 8 \\
8 & 8 & 8 & 8 & 8 & 8 & 8 \\
8 & 8 & 8 & 8 & 8 & 8 & 8 \\
\hline
\end{tabular}

FIG. 2. Flat substrate decorated with regularly spaced elevated ridges, each composed of spheres fixed in a row. In this substrate, the ridge spacing is 5 (in units of the Lennard-Jones diameter of the spheres). 


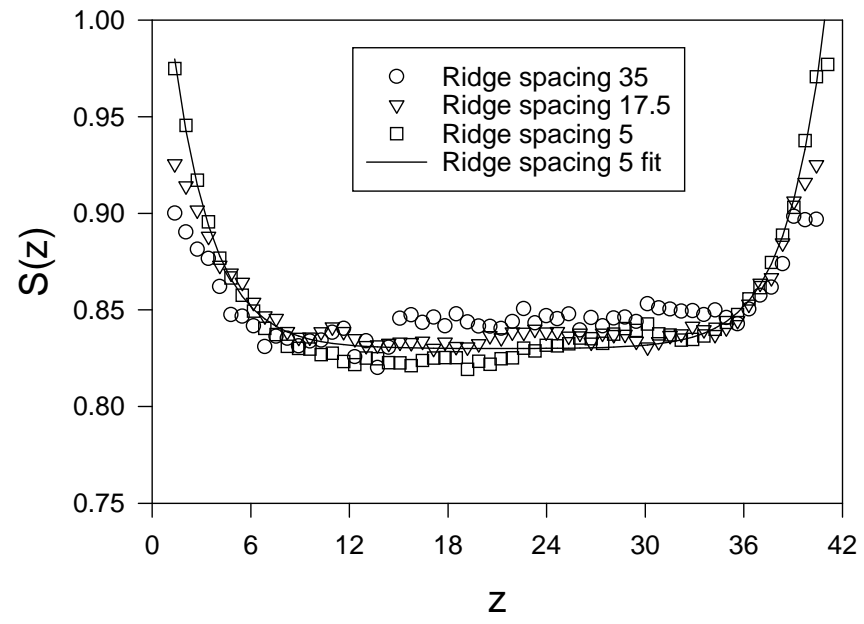

FIG. 3. Profile of the nematic order parameter $S(z)$ as a function of $z$, the distance across the thickness of the cell, for simulation runs with three different ridge spacings on the cell surfaces. Note the enhanced order near the surfaces.

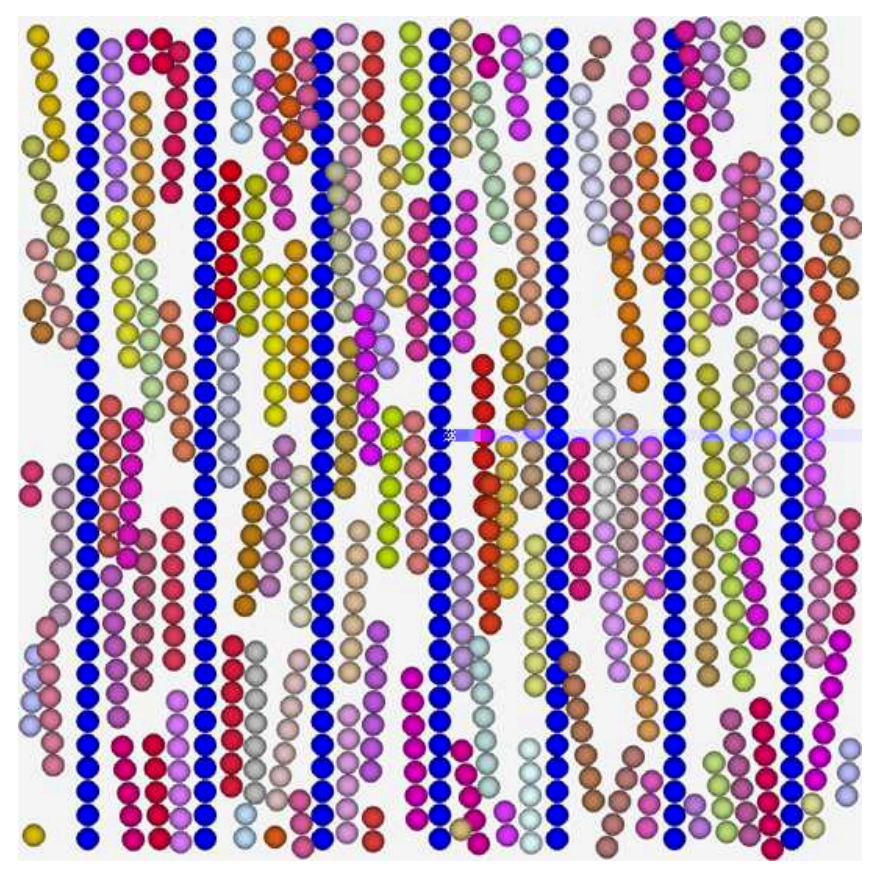

FIG. 4. Snapshot of the molecular configuration near a substrate (with ridge spacing of 5 units). The long vertical rows of spheres are the ridges, while the seven-sphere rods are the molecules. Although the bulk phase is nematic, the region near the substrate shows a local tendency to smectic ordering in the direction along the ridges.

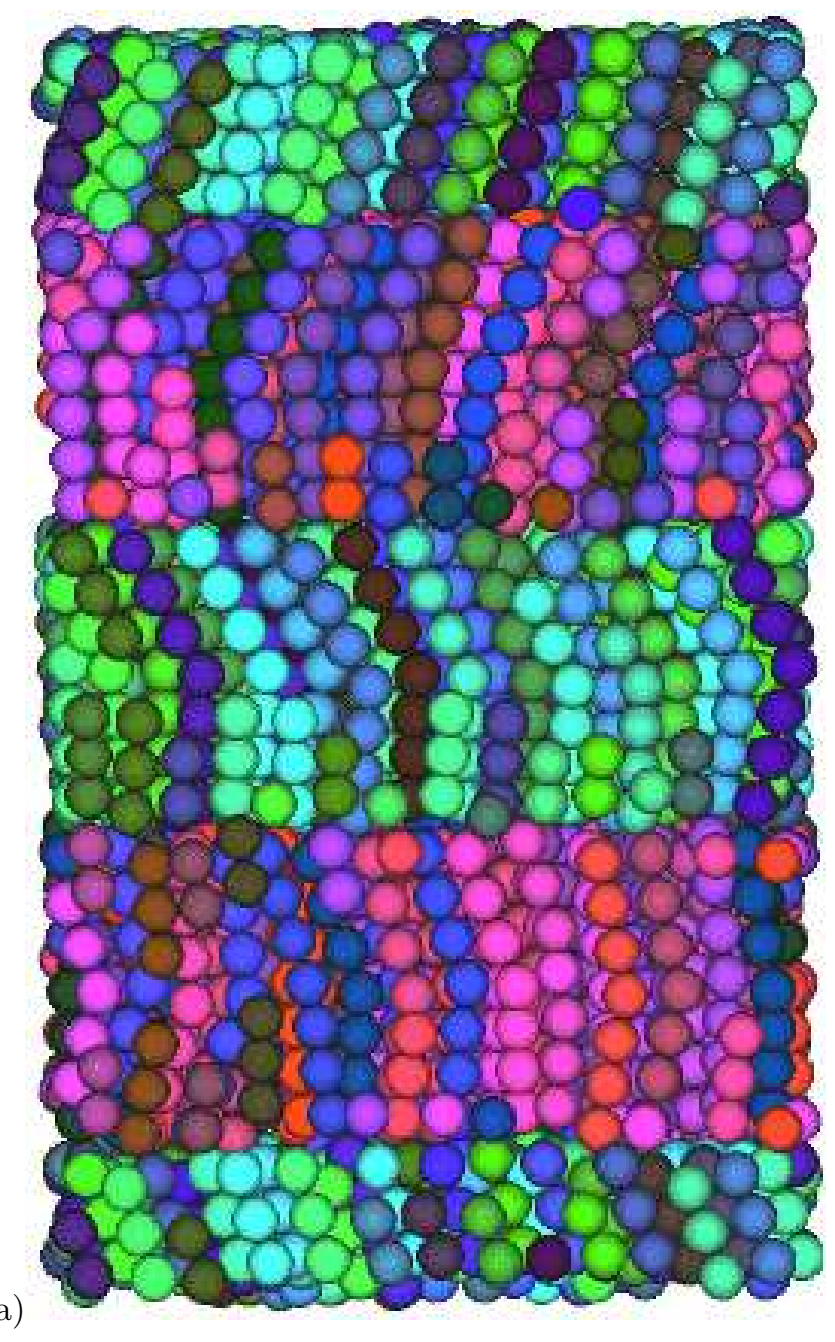

(a)

(b)

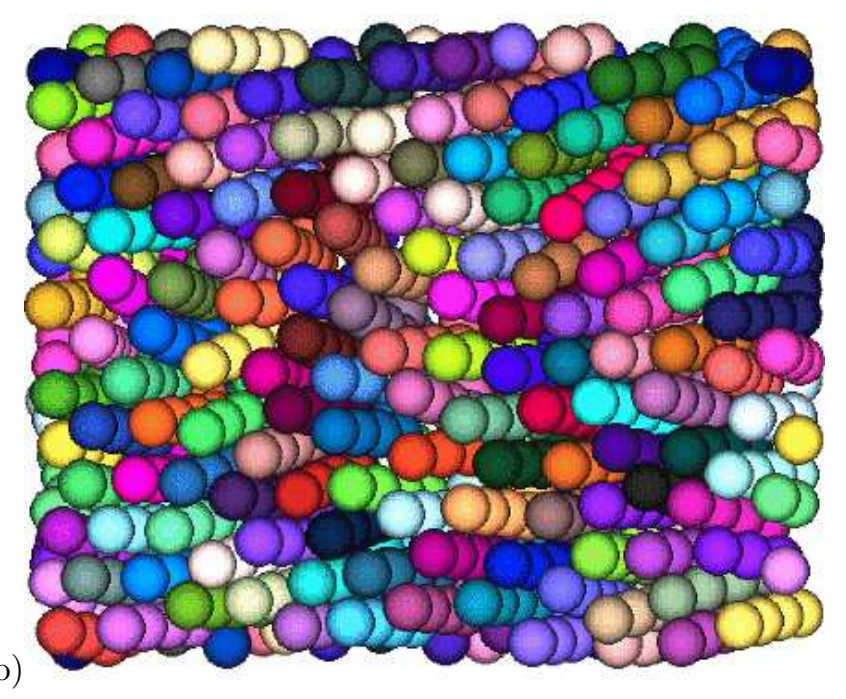

FIG. 5. Snapshot of the configuration of bent-core molecules in a chiral crystalline phase. (a) Side view. (b) Top view of a single layer. 
TABLE I. Fit parameters for the simulations of nematic alignment at three different ridge spacings.

\begin{tabular}{clc}
\hline \hline Ridge spacing & $A$ & $\xi$ \\
\hline 5.0 & 0.175 & 2.3 \\
17.5 & 0.12 & 2.5 \\
35.0 & 0.08 & 2.1 \\
\hline \hline
\end{tabular}

